\title{
Optimal Solar Sail Trajectories for Missions to the Outer Solar System
}

\author{
Bernd Dachwald* \\ German Aerospace Center (DLR), Cologne, Germany
}

\begin{abstract}
Although the solar radiation pressure decreases with the square of solar distance, solar sails enable missions to the outer solar system and even beyond. For such missions, the solar sail may gain a large amount of energy by first making one or more close approaches to the sun. Within this paper, optimal trajectories for solar sail missions to the outer planets and into near interstellar space $(200 \mathrm{AU})$ are presented, both for ideal and for non-ideal sails. Thereby, also near/medium-term solar sails with a relatively moderate performance are considered. The minimal flight time to the outer solar system depends not only on the lightness of the solar sail, but also on the allowed minimal solar distance. The solar distance, however, is limited by the temperature limit of the sail film. Within this paper, it is demonstrated that faster trajectories can be obtained for a given sail temperature limit, if not - as usual - the allowed minimal solar distance but the allowed maximal sail temperature is directly used as an optimization constraint. Although, especially for moderate-performance solar sails, the topology of optimal trajectories becomes quite sophisticated, the required flight times and and the achieved solar system escape velocities obey very simple laws.
\end{abstract}

\section{Introduction}

Utilizing solely the freely available solar radiation pressure for propulsion, solar sails enable a wide range of high- $\Delta V$ missions, many of which are difficult or even impossible to accomplish with any other type of conventional propulsion system. Solar sails enable even missions to the outer solar system and beyond, despite the fact that the solar radiation pressure decreases with the square of the sun-sail distance. For such missions, the solar sail may gain a large amount of energy by first making one or more close approaches to the sun, thereby performing a so-called single or multiple 'solar photonic assist' (SPA) maneuver, turning the trajectory into a hyperbolic one. ${ }^{1-3}$ Within this paper, optimal trajectories for solar sail missions to the outer planets and into near interstellar space $(200 \mathrm{AU})$ are presented, both for ideal and for non-ideal sails. It will be shown that - even for near/medium-term solar sails with relatively moderate performance - such SPA trajectories allow reasonable transfer times, without the need to perform any gravity assist maneuver. Nevertheless, without the use of additional propulsive devices and/or an aerocapture maneuver at the target, only fast flybys can be accomplished due to the associated large hyperbolic excess velocities. The minimal flight time to an outer solar system target depends not only on the lightness of the solar sail, but also on the allowed minimal solar distance: the smaller the minimal solar distance, the larger the amount of energy that can be gained during a solar approach. The minimal solar distance, however, is limited by the temperature limit of the sail film. In previous papers by SAUER ${ }^{1}$ and LEIPOLD, ${ }^{2,3}$ the allowed minimal solar distance was used as a path constraint for trajectory optimization of ideal sails, with the argument that such a constraint enforces that some sail temperature limit will not be exceeded during the closest solar approach. The sail temperature, however, depends not only on the solar distance but also on the sail attitude. Within this paper, it is demonstrated that faster trajectories can be obtained for a given sail temperature limit, if not the allowed minimal solar distance but the allowed maximal sail temperature is directly used as a path constraint for optimization. For the calculation of near-globally optimal trajectories, evolutionary neurocontrol is used, a method that is based on artificial neural networks and evolutionary algorithms. ${ }^{4-6}$

* Research Engineer, Institute of Space Simulation, Linder Hoehe, 51147 Cologne, Germany, Phone: +49-2203-601 3001, Fax: +49-2203-601 2352, E-Mail: bernd.dachwald@dlr.de, AIAA Member, AAS Member 


\section{Solar Sail Force Models}

For describing the solar radiation pressure (SRP) force exerted on a solar sail, it is convenient to introduce two unit vectors. The first one is the sail normal vector $\mathbf{n}$, which is perpendicular to the sail surface and always directed away from the sun. Its direction, which describes the sail attitude, is expressed by the sail clock angle $\alpha$ and the sail cone angle $\beta$ (figure $1(\mathrm{a})$ ). The second unit vector is the thrust unit vector $\mathbf{f}$, which points always along the direction of the SRP force. Its direction is described likewise by the thrust clock angle $\gamma$ and the thrust cone angle $\delta$ (figure 1(b)).

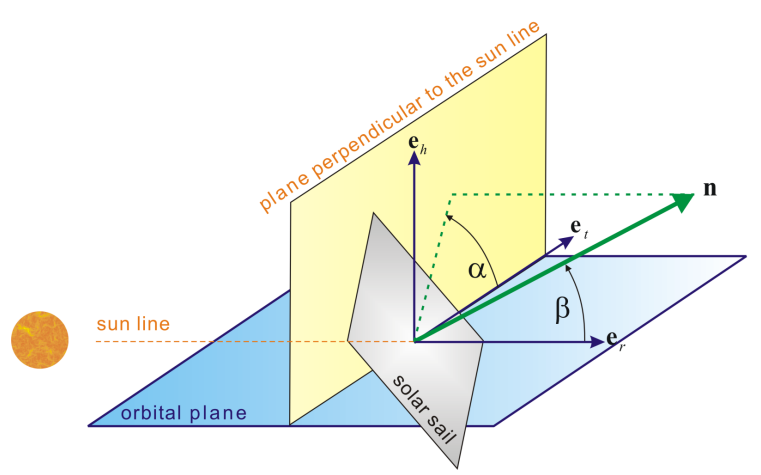

(a)

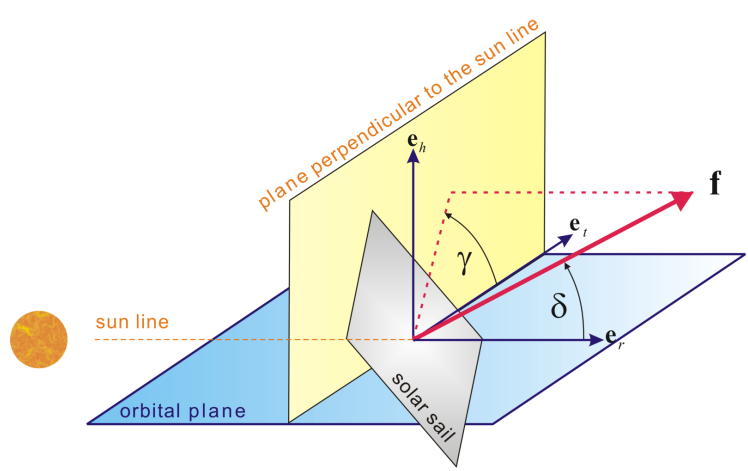

(b)

Figure 1. Definition of the sail normal vector (a) and the thrust unit vector (b)

At a distance $r$ from the sun, the SRP is

$$
P=\frac{S_{0}}{c}\left(\frac{r_{0}}{r}\right)^{2}=4.563 \frac{\mu \mathrm{N}}{\mathrm{m}^{2}} \cdot\left(\frac{r_{0}}{r}\right)^{2}
$$

where $S_{0}=1368 \mathrm{~W} / \mathrm{m}^{2}$ is the solar constant, $c$ is the speed of light in vacuum, and $r_{0}=1 \mathrm{AU}$. For the optical characteristics of a solar sail, different assumptions can be made, which result in different models for the magnitude and direction of the SRP force acting on the sail. The most simple model assumes an ideally reflecting sail surface. The SRP force on an ideal sail of area $A$ is

$$
\mathbf{F}_{\mathrm{SRP}}=2 P A \cos ^{2} \beta \mathbf{n}
$$

Thus, the SRP force it is always along the direction of the sail normal vector, $\mathbf{f}=\mathbf{n}$.

Another SRP force model that is widely encountered uses an overall sail efficiency factor $\eta \leq 1$ with the intention of describing the non-ideal reflectivity of the sail. Using this factor, the SRP force acting on the sail is

$$
\mathbf{F}_{\mathrm{SRP}}=2 \eta P A \cos ^{2} \beta \mathbf{n}
$$

Also for this model, the SRP force is always along the direction of the sail normal vector, $\mathbf{f}=\mathbf{n}$. Therefore, this model also describes a specularly reflecting sail surface, where the angle of incidence is equal to the angle of reflection. From the perspective of trajectory analysis, this models is equivalent to the ideal sail model because a decrease of $\eta$ can always be offset with an inversely proportional increase of $A$. Therefore, this model is not further considered here.

Because the surface of a real solar sail is not a specular reflector, a thorough trajectory simulation must consider the optical characteristics of the real sail film, as they can be parameterized by the absorption coefficient $\alpha$, the reflection coefficient $\rho$, the transmission coefficient $\tau$, and the emission coefficient $\varepsilon$, with the constraint $\alpha+\rho+\tau=1$. The reflection coefficient can be further divided into a coefficient for specular reflection $\rho_{s}$, a coefficient for diffuse reflection $\rho_{d}$, and a coefficient for back reflection $\rho_{b}$, with the constraint $\rho_{s}+\rho_{d}+\rho_{b}=\rho$. The non-ideal solar sail force model used within this paper considers the optical parameters $\mathcal{P}=\left\{\alpha, \rho_{s}, \rho_{d}, \rho_{b}, \tau, \varepsilon_{\mathrm{f}}, \varepsilon_{\mathrm{b}}\right\}$ of a sail film that is aluminum-coated on the front side (emissivity $\varepsilon_{\mathrm{f}}=0.05$ ) and chromium-coated on the back side (emissivity $\varepsilon_{\mathrm{b}}=0.55$ ) to keep the sail temperature within moderate 
limits $^{\mathrm{a}}$. Using the non-ideal solar sail force model, the SRP force has a component

$$
F_{\perp}=2 P A q_{\perp}(\beta, \mathcal{P})
$$

perpendicular to the sail surface and a component

$$
F_{\|}=2 P A q_{\|}(\beta, \mathcal{P})
$$

parallel to the sail surface. Using the optical parameters for an $\mathrm{Al} \mid \mathrm{Cr}$-coated sail, ${ }^{7}$ one gets

$$
\begin{aligned}
q_{\perp}\left(\beta, \mathcal{P}_{\mathrm{Al} \mid \mathrm{Cr}}\right) & =0.9136 \cos ^{2} \beta-0.005444 \cos \beta \\
q_{\|}\left(\beta, \mathcal{P}_{\mathrm{Al} \mid \mathrm{Cr}}\right) & =0.0864 \sin \beta \cos \beta
\end{aligned}
$$

The SRP force may be written as

$$
\mathbf{F}_{\mathrm{SRP}}=\sqrt{F_{\perp}^{2}+F_{\|}^{2}} \mathbf{f}
$$

and by defining

as

$$
Q^{2}(\beta, \mathcal{P})=\sqrt{q_{\perp}^{2}(\beta, \mathcal{P})+q_{\|}^{2}(\beta, \mathcal{P})}
$$

$$
\mathbf{F}_{\mathrm{SRP}}=2 P A Q^{2}(\beta, \mathcal{P}) \mathbf{f}
$$

Thus, the SRP force is not along the direction of the sail normal vector (except for $\beta=0$ ). The angle between $\mathbf{n}$ and $\mathbf{f}$ is

$$
\varepsilon=\arctan \left(q_{\|} / q_{\perp}\right)
$$

The following performance parameters are commonly used to describe the lightness of solar sails: The sail assembly loading is defined as the mass of the sail assembly (the sail film and the required structure for storing, deploying and tensioning the sail, index 'SA') per unit area:

$$
\sigma_{\mathrm{SA}}=\frac{m_{\mathrm{SA}}}{A}
$$

The sail assembly loading is the key parameter for the efficiency of the solar sail's structural design. The sailcraft loading, the key parameter for the lightness of the entire solar sailcraft, is defined as the specific mass of the sailcraft including the payload (index 'PL'), where the term payload stands for the total sailcraft except the solar sail assembly (i.e., except the propulsion system):

$$
\sigma=\frac{m}{A}=\frac{m_{\mathrm{SA}}+m_{\mathrm{PL}}}{A}=\sigma_{\mathrm{SA}}+\frac{m_{\mathrm{PL}}}{A}
$$

The characteristic acceleration is an equivalent parameter for expressing the lightness of the entire solar sailcraft. It is defined as the SRP acceleration acting on a solar sail that is oriented perpendicular to the sun-line at $1 \mathrm{AU}$ :

$$
a_{c}=\frac{2 S_{0} / c \cdot A \cdot q_{\perp}(0, \mathcal{P})}{m}=\frac{P_{\mathrm{eff}, 0}(\mathcal{P}) \cdot A}{m}=\frac{P_{\mathrm{eff}, 0}(\mathcal{P})}{\sigma_{\mathrm{SA}}+\frac{m_{\mathrm{PL}}}{A}}
$$

For an Al|Cr-coated sail, $P_{\text {eff }, 0}\left(\mathcal{P}_{\mathrm{Al} \mid \mathrm{Cr}}\right)=2 S_{0} / c \cdot q_{\perp}\left(0, \mathcal{P}_{\mathrm{Al} \mid \mathrm{Cr}}\right)=8.288 \mu \mathrm{N} / \mathrm{m}^{2}$.

\section{Solar Sail Orbital Dynamics}

The orbital dynamics of solar sails is in many respects similar to the orbital dynamics of other low-thrust spacecraft. Other low-thrust spacecraft, however, may orient its thrust vector into any desired direction, whereas the thrust vector of a solar sail is constrained to lie on the surface of a bubble that is always directed away from the sun (figure 2). Nevertheless, by controlling the sail orientation relative to the sun, a solar sail can gain orbital angular momentum and spiral outwards - away from the sun - or lose orbital angular momentum and spiral inwards - towards the sun. For SPA trajectories, the minimal flight time depends not only on the lightness of the solar sail but also on minimal solar distance along the trajectory (see figure 4).

\footnotetext{
${ }^{\mathrm{a}}$ as it will become clear later from Eq. (15)
} 


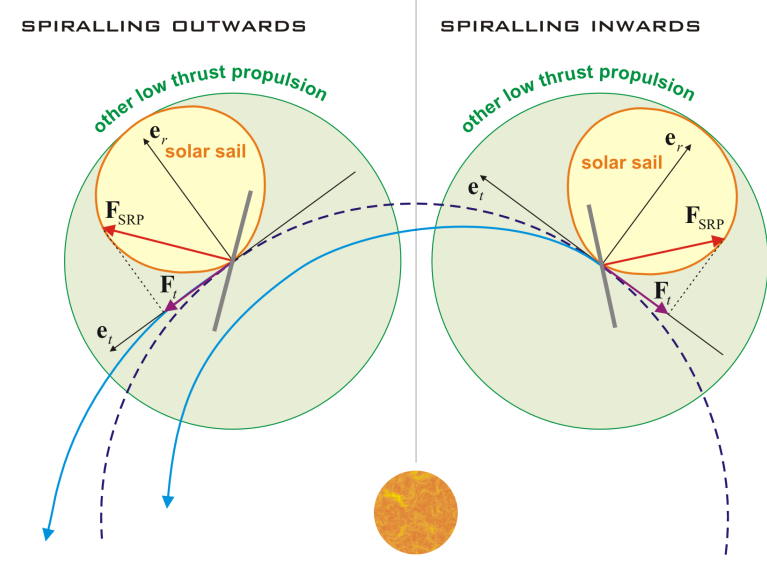

Figure 2. Spiralling towards and away from the sun

The smaller the minimal solar distance, the larger the amount of energy that can be gained during a SPA. The sail's equilibrium temperature at a distance $r$ from the sun is ${ }^{8}$

$$
T=\left(\frac{1-\rho}{\varepsilon_{\mathrm{f}}+\varepsilon_{\mathrm{b}}} \frac{S_{0}}{\sigma} \frac{r_{0}^{2}}{r^{2}} \cos \beta\right)^{1 / 4} \propto \frac{\cos ^{1 / 4} \beta}{r^{1 / 2}}
$$

where $\sigma$ is the Stefan-Boltzmann constant. Therefore, the minimal distance to the sun is - for a given sail attitude - limited by the temperature limit of the sail film. Trajectory optimization for SPA trajectories is exceedingly difficult because one must not only beware of flying too close to the sun but one must also take care that the trajectory becomes not hyperbolic too early, so that no additional energy can be gained. At the same time, one must find the optimal trade-off between the time that is spent within the inner solar system - to gain energy - and the time that is required to fly outwards after the trajectory became hyperbolic.

\section{Evolutionary Neurocontrol: A Smart Global Optimization Method for Solar Sail Trajectories}

Within this paper, evolutionary neurocontrol (ENC) is used for the calculation of near-globally optimal trajectories. This method is based on artificial neural networks (ANNs) and evolutionary algorithms (EAs). ENC attacks trajectory optimization problems from the perspective of artificial intelligence and machine learning. Here, it can only be sketched how this method is used to search optimal solar sail trajectories. The reader who is interested in the details of the method is referred to Refs. 4-6. The problem of searching an optimal solar sail trajectory $\mathbf{x}^{\star}[t]=\left(\mathbf{r}^{\star}[t], \dot{\mathbf{r}}^{\star}[t]\right)$, where ${ }^{\prime}[t]^{\prime}$ denotes the time history of the preceding variable, is equivalent to the problem of searching an optimal sail normal vector history $\mathbf{n}^{\star}[t]$, as it is defined by the optimal time history of the so-called direction unit vector $\mathbf{d}^{\star}[t]$, a unit vector that points along the optimal thrust direction. Within the context of machine learning, a trajectory is regarded as the result of a sail steering strategy $\mathrm{S}$ that maps the problem relevant variables (the solar sail state $\mathbf{x}$ and the target state $\mathbf{x}_{\mathrm{T}}$ ) onto the direction unit vector, $\mathrm{S}:\left\{\mathbf{x}, \mathbf{x}_{\mathrm{T}}\right\} \subset \mathbb{R}^{12} \mapsto\{\mathbf{d}\} \subset \mathbb{R}^{3}$, from which $\mathbf{n}$ is calculated. This way, the problem of searching $\mathbf{x}^{\star}[t]$ is equivalent to the problem of searching (or learning) the optimal sail steering strategy $\mathrm{S}^{\star}$. An ANN may be used as a so-called

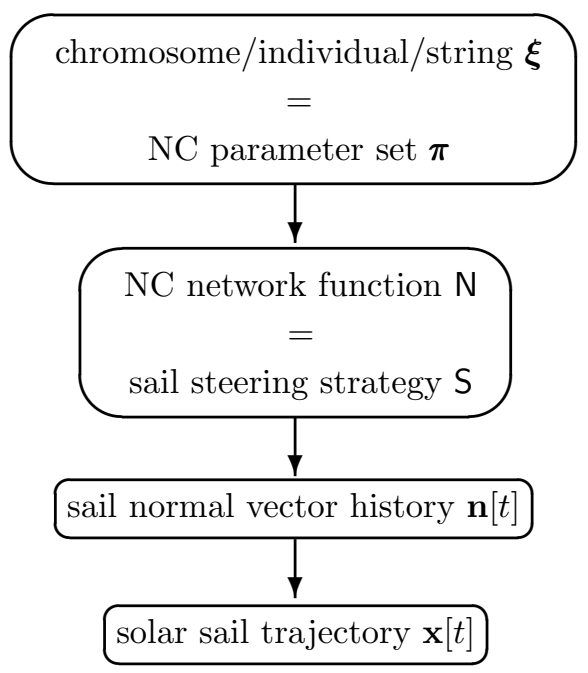

Figure 3. Transformation of a chromosome into a solar sail trajectory neurocontroller $(\mathrm{NC})$ to implement solar sail steering strategies. It can be regarded as a parameterized function $\mathrm{N}_{\boldsymbol{\pi}}$ (the network function) that is - for a given network topology - completely defined by the internal 
parameter set $\boldsymbol{\pi}$ of the ANN. Therefore, each $\boldsymbol{\pi}$ defines a sail steering strategy $\mathrm{S}_{\boldsymbol{\pi}}$. The problem of searching $\mathbf{x}^{\star}[t]$ is therefore equivalent to the problem of searching the optimal NC parameter set $\boldsymbol{\pi}^{\star}$. EAs that work on a population of strings can be used for finding $\boldsymbol{\pi}^{\star}$ because $\boldsymbol{\pi}$ can be mapped onto a string $\boldsymbol{\xi}$ (also called chromosome or individual). The trajectory optimization problem is solved when the optimal chromosome $\xi^{\star}$ is found. Figure 3 sketches the subsequent transformation of a chromosome into a solar sail trajectory. An evolutionary neurocontroller (ENC) is a NC that employs an EA for learning (or breeding) the optimal sail steering strategy. ENC was implemented by the author within a low-thrust trajectory optimization program called InTrance, which stands for Intelligent Trajectory optimization using neurocontroller evolution. InTrance is a smart global trajectory optimization method that requires only the target body/state and intervals for the initial conditions as input to find a near-globally optimal trajectory for the specified problem. It works without an initial guess and does not require the attendance of a trajectory optimization expert.

\section{Results}

All trajectories calculated within this paper assume direct interplanetary insertion of the solar sail with zero hyperbolic excess energy $\left(C_{3}=0 \mathrm{~km}^{2} / \mathrm{s}^{2}\right)$. To find the absolute flight time minima - independent of the actual constellation of Earth and the respective target - no flyby at the target itself but only a crossing of its orbit within a distance of less than $10^{6} \mathrm{~km}$ was required, and InTrance was allowed to vary the launch date within a one year interval. Therefore, the resulting flight times represent lower bounds that are strictly valid only for the optimal constellation of Earth and the respective target. Specific suboptimal launch dates/constellations might yield much longer flight times.

Besides the gravitational forces of all celestial bodies and the SRP force, many disturbing forces - as caused, e.g., by the solar wind and the aberration of solar radiation (POYNTING-ROBERTSON effect) - are influencing the motion of solar sails. Ideally, all these forces have to be considered for a thorough mission analysis. For mission feasibility analysis, however, as it is done within this paper, the following simplifications are valid: (1) the solar sail is moving under the sole influence of solar gravitation and radiation, (2) the sun is a point mass and a point light source, (3) the solar sail attitude can be changed instantaneously, and (4) the optical characteristics of the sail film do not degrade over time.

Later in this paper, it will become necessary to distinguish trajectories, for which the allowed minimal solar distance was limited, from trajectories, for which the allowed maximal sail film temperature was limited. Therefore, let $r_{\text {lim }}$ denote the allowed minimal solar distance (distance limit) and $r_{\min }$ the minimal solar distance along the trajectory, and let $T_{\mathrm{lim}}$ denote the allowed maximal sail film temperature (sail temperature limit) and $T_{\max }$ the maximal sail film temperature along the trajectory. Using this notation, $T_{\max }=$ $T_{\max }\left(r_{\text {lim }}\right)$ (for distance-limited trajectories) and $r_{\min }=r_{\min }\left(T_{\lim }\right)$ (for temperature-limited trajectories).

Before calculating minimal flight times $\mathcal{T}$ and solar system escape velocities ${ }^{\mathrm{b}} v_{\text {esc }}$ for missions to all outer planets and into near interstellar space (200 AU), a Neptune flyby mission will be used to assess the general features of SPA trajectories, to compare different solar sail force models (ideal vs. non-ideal solar sails), and to compare different optimization constraints (limitation of allowed minimal solar distance vs. limitation of allowed maximal sail temperature). Finally, by calculating optimal temperature-limited trajectories for non-ideal solar sails, it will be investigated how the minimal flight time, the solar system escape velocity, and the topology of the trajectory (e.g., the number of SPAs) vary for a wide range of characteristic accelerations and sail temperature limits.

\section{A. Dependency of Minimal Flight Time on Lightness and Minimal Solar Distance for Ideal Solar Sails}

For a Neptune flyby, InTrance was used to calculate minimal flight times for ideal solar sails with different characteristic accelerations $\left(0.5 \mathrm{~mm} / \mathrm{s}^{2} \leq a_{c} \leq 2.0 \mathrm{~mm} / \mathrm{s}^{2}\right)$ and different solar distance limits $(0.1 \mathrm{AU} \leq$ $\left.r_{\lim } \leq 0.5 \mathrm{AU}\right)$. Figure 4 shows the results. As expected, the trajectories are faster for lighter solar sails and for sails that are allowed to make closer approaches to the sun. Interestingly, figure 4 shows that $\mathcal{T}\left(r_{\lim }\right)$ obeys approximately a linear law for all three values of $a_{c}$,

$$
\mathcal{T}\left(a_{c}, r_{\lim }\right) \approx c_{1}\left(a_{c}\right) r_{\lim }+c_{2}\left(a_{c}\right) .
$$

\footnotetext{
${ }^{\mathrm{b}} v_{\text {esc }}=\sqrt{ }\left(v_{f}^{2}-2 \mu / r_{f}\right)$, where $v_{f}$ is the final velocity, $r_{f}$ is the final solar distance, and $\mu$ is the sun's gravitational constant
} 


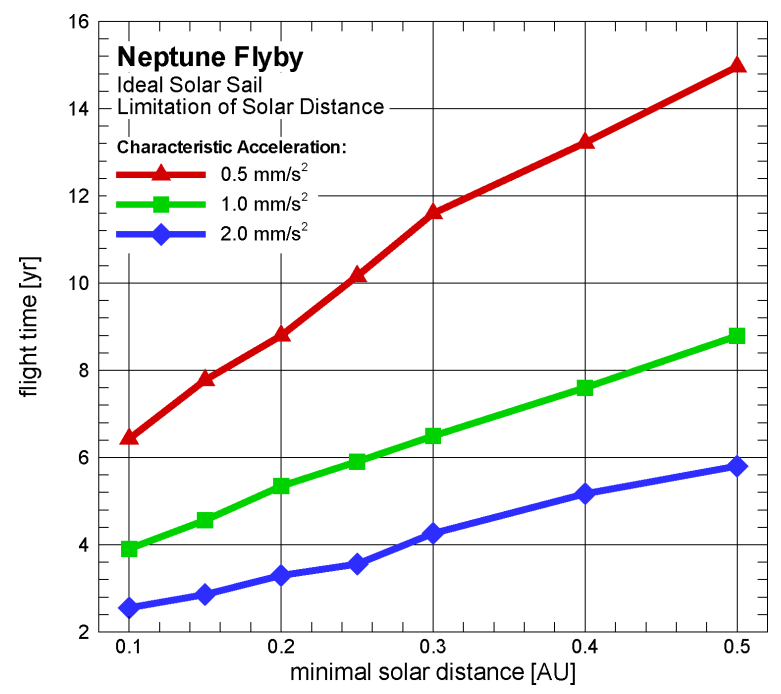

Figure 4. Minimal flight time over characteristic acceleration for different distance-limits $r_{\text {lim }}$ (ideal sail)

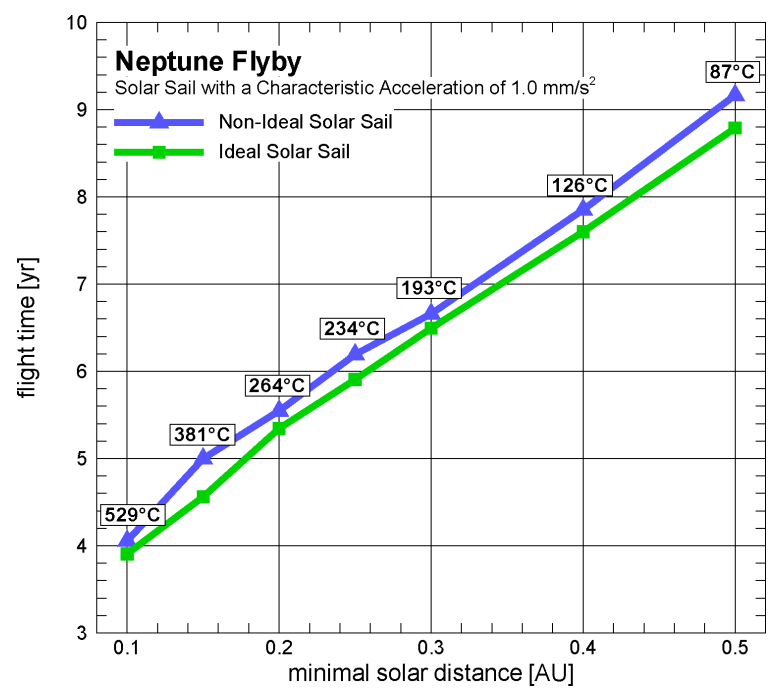

Figure 5. Comparison of minimal flight times for ideal and non-ideal solar sails $\left(a_{c}=1.0 \mathrm{~mm} / \mathrm{s}^{2}\right)$

Figure 6 shows optimal Neptune flyby trajectories for four different characteristic accelerations, the allowed minimal solar distance being limited to $r_{\mathrm{lim}}=0.1 \mathrm{AU}$ in all four cases. One can see that more and more SPAs are required as the characteristic acceleration of the sail decreases. The optimal trajectory for the lightest solar sail $\left(a_{c}=2.0 \mathrm{~mm} / \mathrm{s}^{2}\right)$ makes only a single SPA, whereas the optimal trajectory for the heaviest solar sail $\left(a_{c}=0.5 \mathrm{~mm} / \mathrm{s}^{2}\right)$ requires four SPAs to reach Neptune in minimal time. The heavier the solar sail is, the larger is also the fraction of flight time that must be spent in the inner solar system for gaining orbital energy ( $30.1 \%$ of total flight time for $a_{c}=2.0 \mathrm{~mm} / \mathrm{s}^{2}, 33.5 \%$ for $a_{c}=1.0 \mathrm{~mm} / \mathrm{s}^{2}, 39.9 \%$ for $a_{c}=0.75 \mathrm{~mm} / \mathrm{s}^{2}$, and $44.5 \%$ for $\left.a_{c}=0.5 \mathrm{~mm} / \mathrm{s}^{2}\right)$.

\section{B. Ideal vs. Non-Ideal Solar Sails}

In the previous section, minimal flight times have been presented for ideal solar sails because, to the authors knowledge, all previous solar sail trajectory analyses for solar system escape missions assume ideal reflectivity of the sail. A real solar sail, however, is not an ideal reflector and a thorough trajectory analysis must take into account the optical characteristics of the real sail film. ${ }^{9}$ In Figure 5, flight times are compared for an 


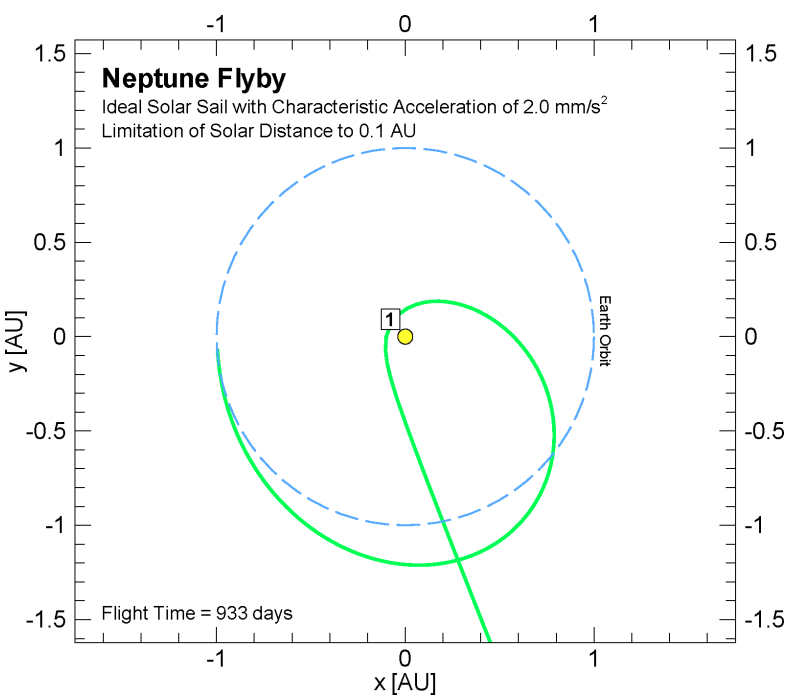

(a) $a_{c}=2.0 \mathrm{~mm} / \mathrm{s}^{2}$, one solar photonic assist

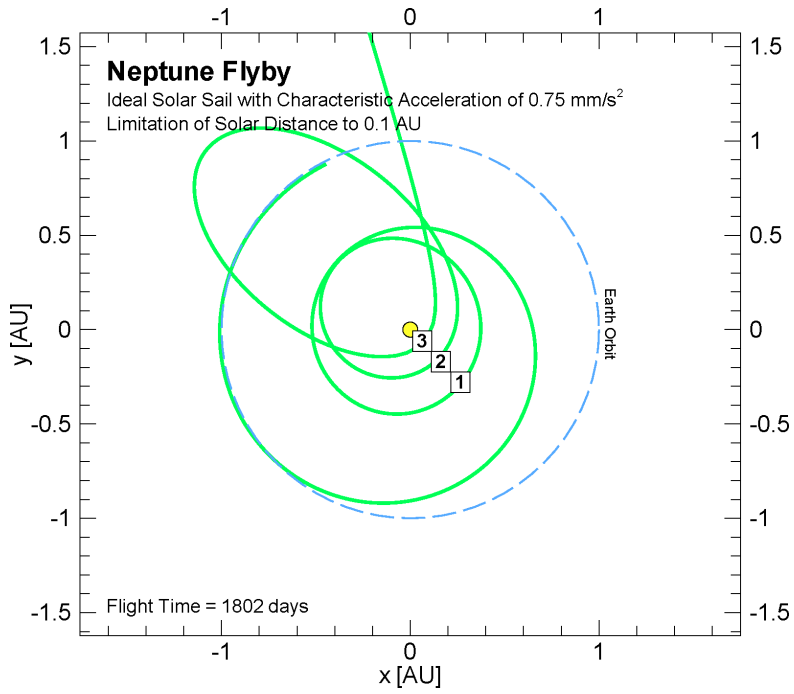

(c) $a_{c}=0.75 \mathrm{~mm} / \mathrm{s}^{2}$, three solar photonic assists

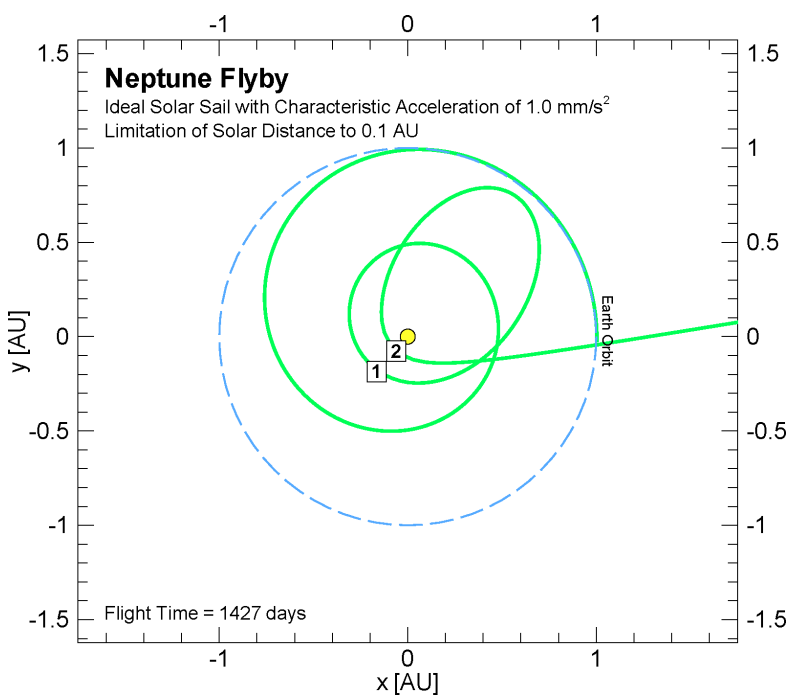

(b) $a_{c}=1.0 \mathrm{~mm} / \mathrm{s}^{2}$, two solar photonic assists

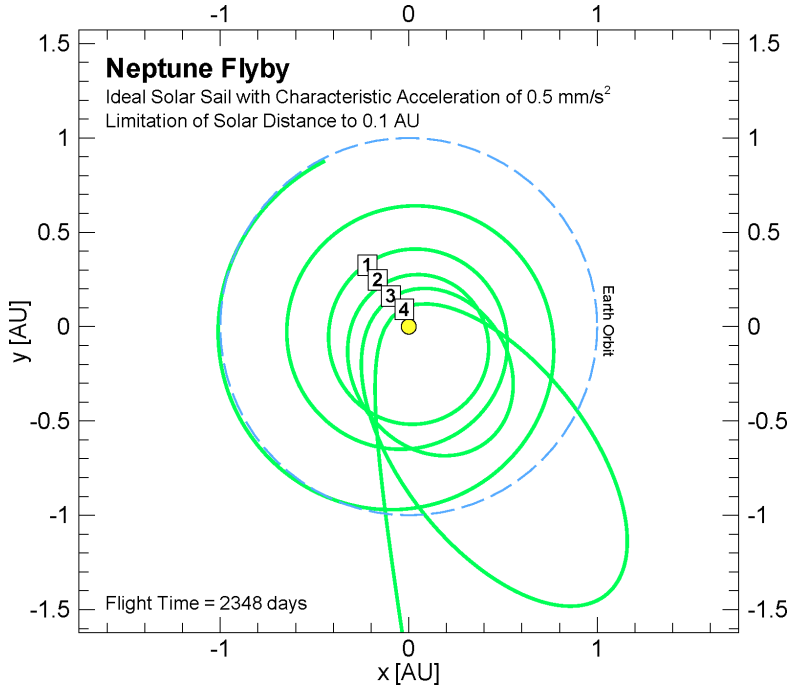

(d) $a_{c}=0.5 \mathrm{~mm} / \mathrm{s}^{2}$, four solar photonic assists

Figure 6. Topology of optimal Neptune flyby trajectories for different characteristic accelerations (ideal sail, $\left.r_{\lim }=0.1 \mathrm{AU}\right)$

ideal and a non-ideal solar sail with $a_{c}=1.0 \mathrm{~mm} / \mathrm{s}^{2}$. Figure 5 shows that the flight times are about $5 \%$ longer, if the non-ideal reflectivity of the sail is taken into account.

\section{Distance-Limited vs. Temperature-Limited Trajectories}

In previous papers by SAUER ${ }^{1}$ and LEIPOLD, ${ }^{2,3} r_{\text {lim }}$ was used as a path constraint for the trajectory optimization of ideal solar sails, with the argument that such a constraint enforces that some $T_{\text {lim }}$ will not be exceeded during the closest solar approach. ${ }^{c}$ According to Eq. (15), however, the temperature at a given solar distance $r$ depends also on the light incidence angle $\beta$. It might therefore yield faster trajectories for a given $T_{\text {lim }}$, if not $r_{\lim }$ but $T_{\text {lim }}$ is directly used as a path constraint. This can be realized by constraining the sail attitude so that the light incidence angle can not become smaller than the critical one, $\beta>\beta_{\lim }\left(r, T_{\lim }\right)$, where $T_{\lim }$ would be exceeded. Figure 7 shows two exemplary trajectories, one distance-limited (figure $7(\mathrm{a})$ ) and one temperature-limited (figure $7(\mathrm{~b})$ ). By keeping $\beta$ large enough during the closest approach, the temperature-limited trajectory approaches the sun closer while maintaining the sail temperature below the

\footnotetext{
${ }^{\mathrm{c}}$ although - strictly - the temperature of an ideal sail is always $0 \mathrm{~K}$ according to equation (15)
} 


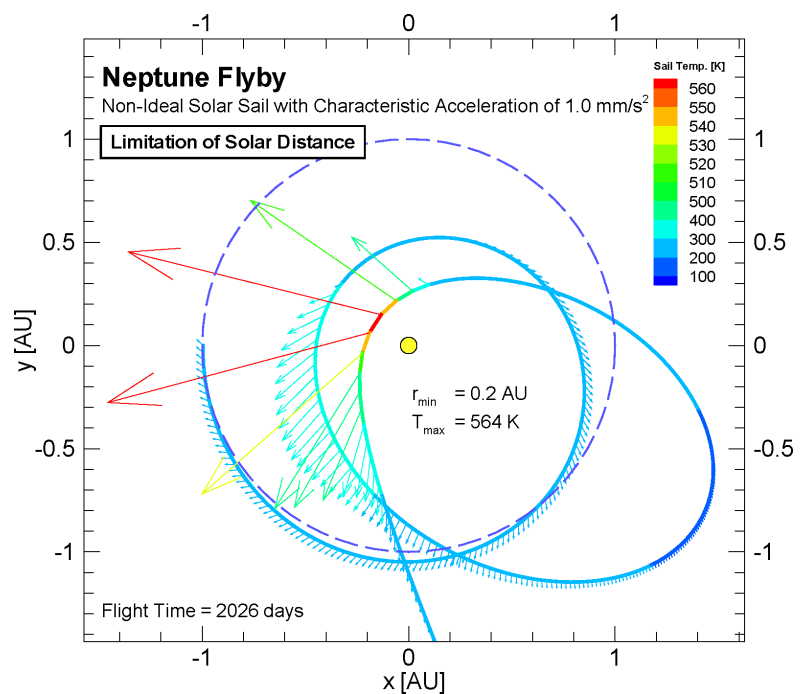

(a) Distance-limited trajectory

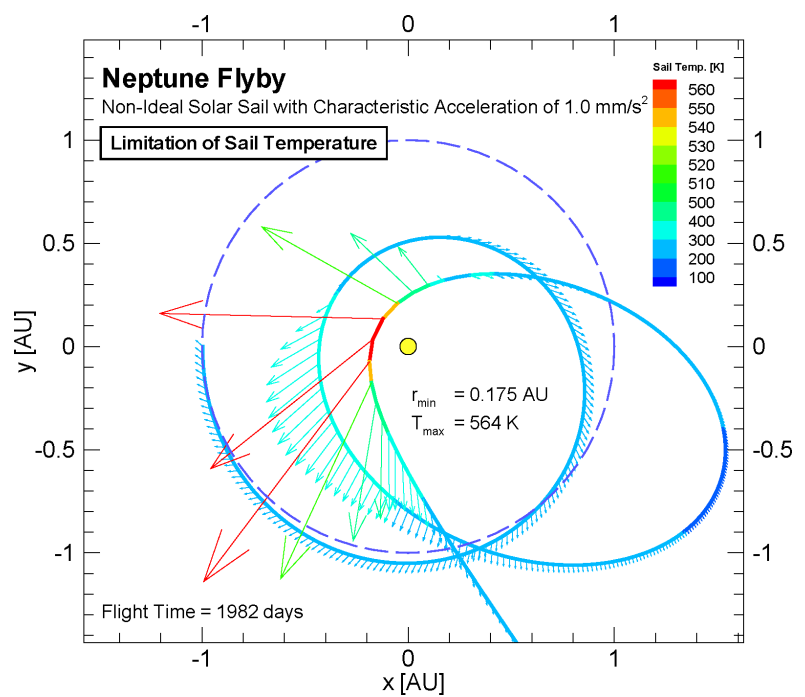

(b) Temperature-limited trajectory

Figure 7. Optimal Neptune flyby trajectories for different optimization constraints $\left(a_{c}=1.0 \mathrm{~mm} / \mathrm{s}^{2}\right)$

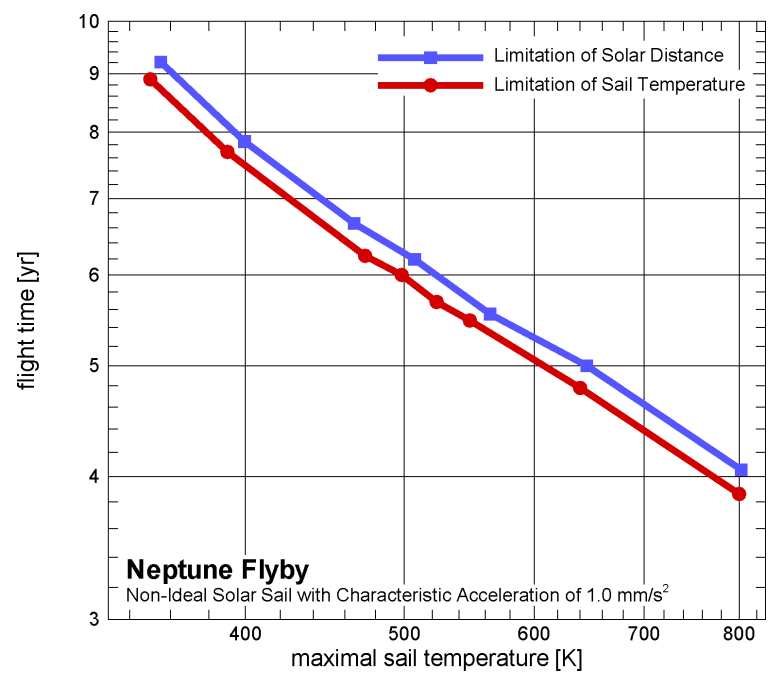

Figure 8. Flight time over maximum sail temperature for distance-limited and temperature-limited optimal trajectories

temperature that is given by the distance-limited trajectory (so that $T_{\max }$ is the same for both trajectories). The resulting trajectory is faster than the distance-limited one. Figure 8 shows that for a given $T_{\text {lim }}$, optimal temperature-limited trajectories are on average about $5 \%$ faster than optimal distance-limited ones. Interestingly, figure 8 shows that $\mathcal{T}$ and $T_{\max }$ are nearly inversely proportional for both curves,

$$
\mathcal{T}\left(T_{\max }\right) \approx c_{3} / T_{\max }
$$

\section{Minimal Flight Times to the Outer Planets and Near Interstellar Space}

Figure 9 shows the minimal flight times $\mathcal{T}$ and the achieved hyperbolic escape velocities $v_{\text {esc }}$ for optimal temperature-limited flyby trajectories to the outer planets and to $200 \mathrm{AU}$ using a non-ideal solar sail. The sail film temperature was limited to $T_{\text {lim }}=240^{\circ} \mathrm{C}$. Interestingly, figure 9 shows that $\mathcal{T}\left(a_{c}\right)$ obeys nearly a potential law for all targets,

$$
\mathcal{T}\left(\text { Target }, a_{c}\right) \approx c_{4} \text { (Target) } a_{c}^{c_{5} \text { (Target) }}
$$



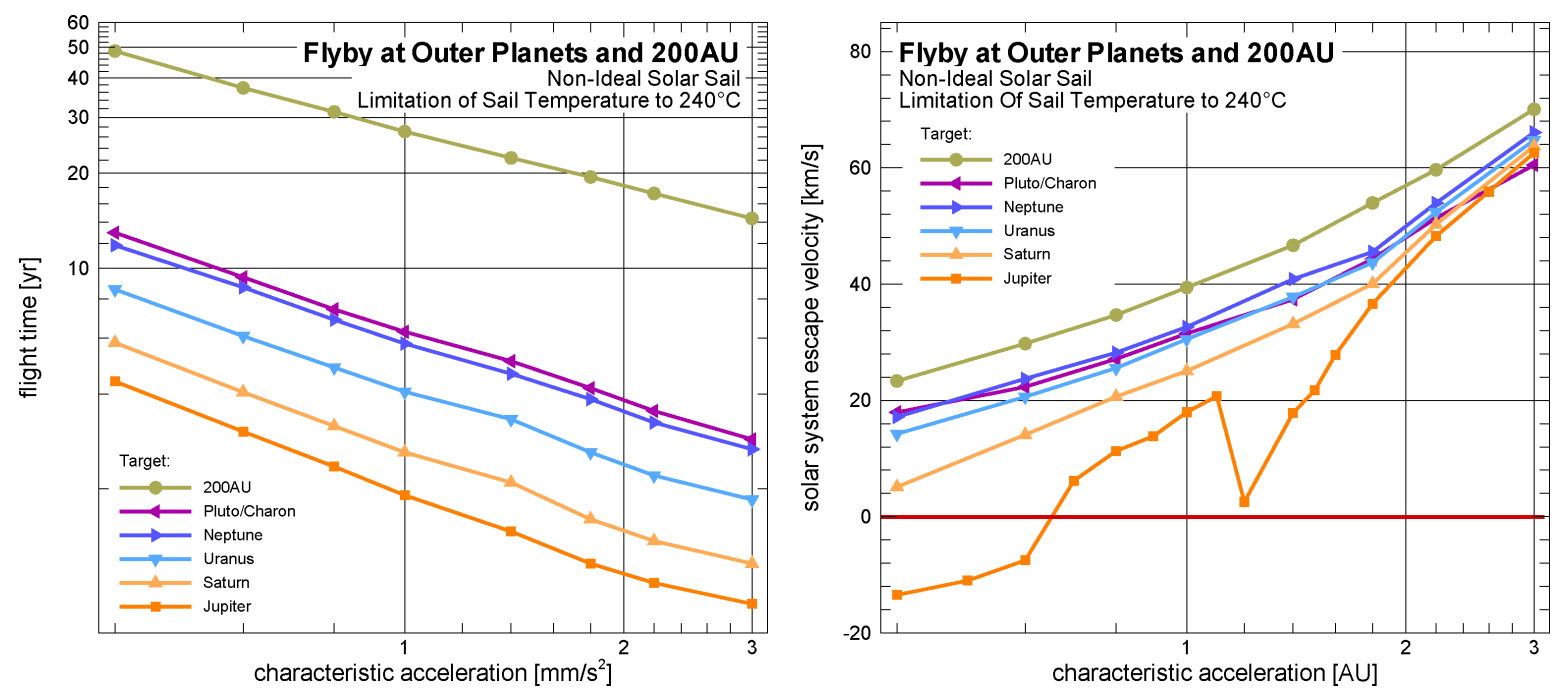

Figure 9. Temperature-limited optimal flyby with non-ideal solar sail

with $-0.827<c_{5}$ (Target) $<-0.601$. The right side of figure 9 shows that $v_{\text {esc }}$ increases for more distant targets because it is beneficial, in this case, to spend more time in the inner solar system to gain more energy. Figure 9 shows that even near-term solar sails $\left(a_{c} \approx 0.4 \mathrm{~mm} / \mathrm{s}^{2}\right)$ are able to reach Uranus within less than 10 years, and that even medium-term solar sails $\left(a_{c} \approx 0.6 \mathrm{~mm} / \mathrm{s}^{2}\right)$ are able to reach Neptune and the inner EDGEWORTH-KUIPER belt within less than 10 years. A more advanced solar sail, $a_{c}=1.4 \mathrm{~mm} / \mathrm{s}^{2}$, can reach $200 \mathrm{AU}$ within less than 25 years. The $v_{\text {esc }}$-curve for Jupiter shows a discontinuity for $1.1 \mathrm{~mm} / \mathrm{s}^{2}<a_{c}<$ $1.2 \mathrm{~mm} / \mathrm{s}^{2}$ that is due to a change in the topology of the optimal trajectory: the optimal trajectories for $a_{c} \gtrsim 1.2 \mathrm{~mm} / \mathrm{s}^{2}$ perform only a single SPA whereas the optimal trajectory for $a_{c}=1.1 \mathrm{~mm} / \mathrm{s}^{2}$ performs a double SPA. Thereby, the second SPA is not performed in order to maximize $v_{\text {esc }}$. Such a second SPA would require a larger aphelion, but more time would be required to reach this aphelion than would be saved due to the larger escape velocity. The $v_{\mathrm{esc}}$-curves for more distant targets indicate no discontinuity because the double SPAs are performed in order to maximize $v_{\text {esc }}$. It is also interesting to note that the time-optimal Jupiter flyby trajectory for $a_{c} \lesssim 0.65 \mathrm{~mm} / \mathrm{s}^{2}$ is not a hyperbolic but an elliptic one $\left(v_{\text {esc }}<0 \mathrm{~km} / \mathrm{s}\right)$. Because of the interesting features of Jupiter's $v_{\text {esc }}$-curve, optimal trajectories have been calculated for a wider range of characteristic accelerations $\left(0.4 \mathrm{~mm} / \mathrm{s}^{2} \leq a_{c} \leq 8.0 \mathrm{~mm} / \mathrm{s}^{2}\right)$ and for three different sail temperature limits $\left(200^{\circ} \mathrm{C}, 240^{\circ} \mathrm{C}\right.$, and $\left.280^{\circ} \mathrm{C}\right)$. The results for those calculations, shown in figure 10 , will be described in the next section. A translation of the sail temperature limits into sail film materials is not within the scope of this paper because the allowed maximal sail film temperature depends not only on the film material but also on the sail design (stresses, wrinkles, etc.).

\section{E. Dependency of Minimal Flight Time on Sail Temperature Limit for Non-Ideal Solar Sails}

Figure 10 shows for three different sail temperature limits $\left(200^{\circ} \mathrm{C}, 240^{\circ} \mathrm{C}\right.$, and $\left.280^{\circ} \mathrm{C}\right)$ the minimal flight times and the achieved escape velocities for temperature-limited optimal flyby trajectories to Jupiter. One can see that there is a discontinuity - like the one observed in figure 9 - for all three temperature-limits, but at different characteristic accelerations. For a higher $T_{\text {lim }}$, the optimal trajectory employs a single SPA down to lower characteristic accelerations, until a double SPA is optimal. If one considers also very large characteristic accelerations, a second discontinuity occurs in the range $6 \mathrm{~mm} / \mathrm{s}^{2} \lesssim a_{c} \lesssim 7 \mathrm{~mm} / \mathrm{s}^{2}$. This discontinuity is again due to a change in the topology of the optimal trajectory: the optimal trajectories for $a_{c} \gtrsim 7 \mathrm{~mm} / \mathrm{s}^{2}$ do not make a SPA because a SPA would take longer than a direct trajectory to Jupiter. Direct trajectories are feasible in this performance regime because the maximal acceleration of the solar sail is larger than the gravitational attraction of the sun $\left(a_{0}=5.93 \mathrm{~mm} / \mathrm{s}^{2}\right.$ at $\left.1 \mathrm{AU}\right)$. The relation $a_{c} / a_{0}$ is independent of solar distance because both vary with $1 / r^{2}$. For $a_{c}>a_{0}$, the sail's acceleration capability is more than enough to cancel solar gravitation, so that the solar sail does not need to orbit but is able to fly directly away from the sun. Interestingly, figure 10 shows also that the flight time does not depend considerably on $T_{\text {lim }}$ or $v_{\text {esc }}$ but only on $a_{c}$. Within the low/medium-performance regime $\left(a_{c} \lesssim 2.0 \mathrm{~mm} / \mathrm{s}^{2}\right)$ 

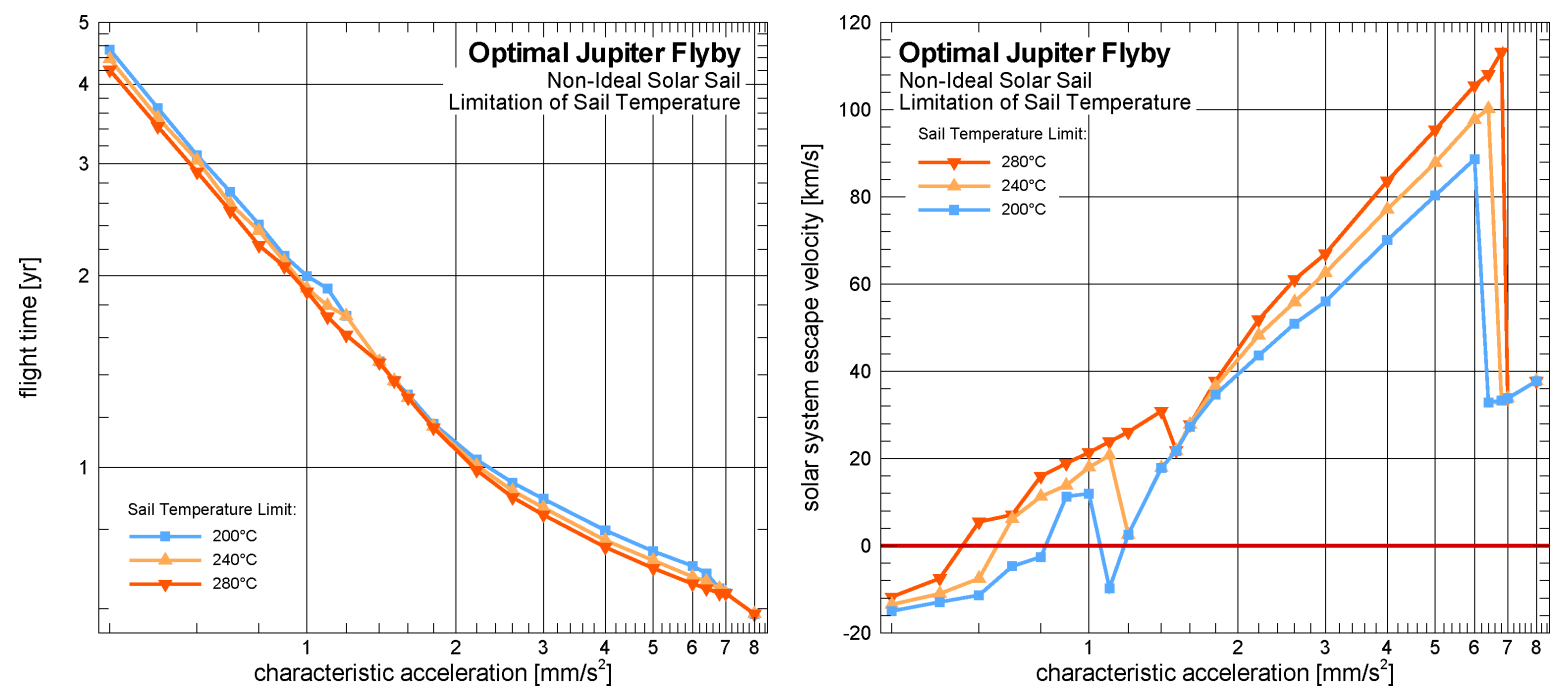

Figure 10. Temperature-limited optimal Jupiter flyby with non-ideal solar sail
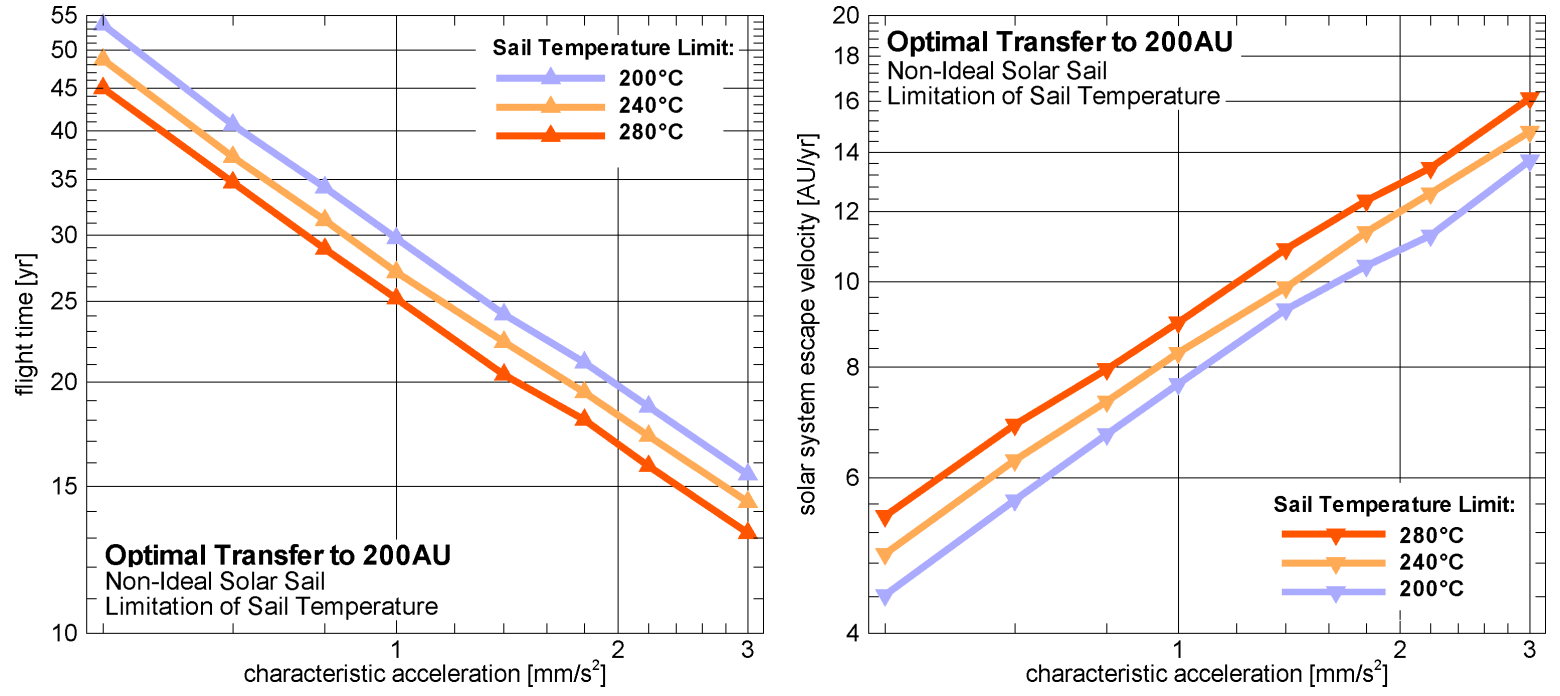

Figure 11. Temperature-limited optimal transfer to $200 \mathrm{AU}$ with non-ideal solar sail

and the very high-performance regime $\left(a_{c} \gtrsim 3.0 \mathrm{~mm} / \mathrm{s}^{2}\right), \mathcal{T}\left(a_{c}\right)$ obeys nearly a potential law,

$$
\mathcal{T}\left(a_{c}\right) \approx c_{4} a_{c}^{c_{5}}
$$

( $c_{5}$ being different for both regimes), with a transition regime, where the $\mathcal{T}\left(a_{c}\right)$-curve bends.

Figure 11 shows for three different sail temperature limits $\left(200^{\circ} \mathrm{C}, 240^{\circ} \mathrm{C}\right.$, and $\left.280^{\circ} \mathrm{C}\right)$ the minimal flight times and the achieved escape velocities for temperature-limited optimal transfers to $200 \mathrm{AU}$. The $v_{\text {esc }}{ }^{-}$ curve does not show the strange features of Jupiter's $v_{\text {esc }}$-curve, because all SPAs are performed in order to maximize $v_{\text {esc }}$. Figure 11 shows that $\mathcal{T}\left(a_{c}\right)$ and $v_{\text {esc }}\left(a_{c}\right)$ obey a potential law for all sail temperature limits,

$$
\mathcal{T}\left(a_{c}, T_{\lim }\right)=c_{4}\left(T_{\lim }\right) a_{c}^{c_{5}}
$$

with $c_{5}=0.543^{ \pm 0.011}$ and

$$
v_{\mathrm{esc}}\left(a_{c}, T_{\mathrm{lim}}\right)=c_{6}\left(T_{\lim }\right) a_{c}^{c_{7}} .
$$

with $c_{7}=-0.605^{ \pm 0.006}$. Figure 11 shows that the minimal flight times depend considerably on the sail temperature limit. For $T_{\lim }=280^{\circ} \mathrm{C}$, a characteristic acceleration of about $1.0 \mathrm{~mm} / \mathrm{s}^{2}$ is required to reach 
$200 \mathrm{AU}$ within 25 years from launch, whereas a characteristic acceleration of about $1.4 \mathrm{~mm} / \mathrm{s}^{2}$ is required for $T_{\text {lim }}=200^{\circ} \mathrm{C}$. Note that the solar sail design parameters can be very sensitive with respect to the characteristic acceleration, as the following example may show: if a solar sail with a sail assembly loading of $\sigma_{\mathrm{SA}}=5 \mathrm{~g} / \mathrm{m}^{2}$ should be used to transport a payload (incl. spacecraft bus) of $m_{\mathrm{PL}}=100 \mathrm{~kg}$ to $200 \mathrm{AU}$, $a_{c}=1.0 \mathrm{~mm} / \mathrm{s}^{2}$ yields - according to Eq. $(14)$ - a sail area of $A=(175 \mathrm{~m})^{2}$, whereas $a_{c}=1.4 \mathrm{~mm} / \mathrm{s}^{2}$ yields a sail area of $A=(330 \mathrm{~m})^{2}$. If the sail size is held at $(175 \mathrm{~m})^{2}$, the payload reduces to $m_{\mathrm{PL}}=28 \mathrm{~kg}$. Another possibility is to decrease the sail assembly loading to $\sigma_{\mathrm{SA}}=2.6 \mathrm{~g} / \mathrm{m}^{2}$, which can only be done with some much more advanced sail fabrication/deployment technology.

\section{Summary and Conclusions}

The material presented within this paper provides trajectory and performance trade-offs for missions to the outer solar system and to near interstellar space (200 AU). A wide range of solar sail performance levels and sail temperature limits has been considered. To the authors knowledge, all previously presented trajectory analyses for solar system escape missions have been carried out for ideal high-performance sails. Within this paper, optimal trajectories have been presented both for ideal and for non-ideal solar sails. Thereby, also near/medium-term solar sails with a relatively moderate performance have been considered. It was shown that a thorough trajectory analysis must consider the non-ideal reflectivity of the solar sail, which yields minimal flight times that are about $5 \%$ longer than those of ideal sails. To the authors knowledge, all previously presented trajectory analyses for solar system escape missions constrain the minimal solar distance, with the argument that such a constraint enforces that some sail temperature limit will not be exceeded during the closest solar approach. The trajectories calculated within this paper demonstrate that faster trajectories can be obtained for a given sail temperature limit, if not the allowed minimal solar distance but the allowed maximal sail temperature is directly used as an optimization constraint. For temperaturelimited trajectories, minimal flight times and solar system escape velocities have been presented for missions to all outer planets and to near interstellar space. Although, especially for moderate-performance solar sails, the topology of optimal trajectories is quite sophisticated, the required flight times and the achieved solar system escape velocities obey very simple laws that are, however, not yet theoretically understood.

\section{References}

\footnotetext{
${ }^{1}$ Sauer, C., "Solar Sail Trajectories for Solar Polar and Interstellar Probe Missions," Astrodynamics 1999, edited by K. Howell, F. Hoots, and B. Kaufman, Vol. 103 of Advances in the Astronautical Sciences, Univelt, Inc., 2000, pp. $547-562$.

${ }^{2}$ Leipold, M. and Wagner, O., "'Solar Photonic Assist' Trajectory Design fo Solar Sail Missions to the Outer Solar System and Beyond," AAS/GSFC 13 ${ }^{\text {th }}$ International Symposium on Space Flight Dynamics, Greenbelt, USA, May 1998.

${ }^{3}$ Leipold, M., "To the Sun and Pluto with Solar Sails and Micro-Sciencecraft," Acta Astronautica, Vol. 45, No. 4-9, 1999, pp. 549-555.

${ }^{4}$ Dachwald, B., Low-Thrust Trajectory Optimization and Interplanetary Mission Analysis Using Evolutionary Neurocontrol, Doctoral thesis, Universität der Bundeswehr München; Fakultät für Luft- und Raumfahrttechnik, 2004.

${ }^{5}$ Dachwald, B., "Optimization of Interplanetary Solar Sailcraft Trajectories Using Evolutionary Neurocontrol," Journal of Guidance, Control, and Dynamics, Vol. 27, No. 1, pp. 66-72.

${ }^{6}$ Dachwald, B., "Evolutionary Neurocontrol: A Smart Method for Global Optimization of Low-Thrust Trajectories," AIAA/AAS Astrodynamics Specialist Conference, Providence, USA, August 2004, AIAA-2004-5405.

${ }^{7}$ Wright, J., Space Sailing, Gordon and Breach Science Publishers, Philadelphia, 1992.

${ }^{8}$ McInnes, C., Solar Sailing. Technology, Dynamics and Mission Applications, Springer-Praxis Series in Space Science and Technology, Springer-Praxis, Berlin, Heidelberg, New York, Chicester, 1999.

${ }^{9}$ Dachwald, B., "Interplanetary Mission Analysis for Non-Perfectly Reflecting Solar Sailcraft Using Evolutionary Neurocontrol," AAS/AIAA Astrodynamics Specialist Conference, Big Sky, USA, August 2003, AAS 03-579.
} 